\title{
The Arithmetic Properties of Lineation in Humanistic Manuscripts
}

The counting of lines is a necessary task that codicologists and cataloguers feel obliged to perform in a diligent way, but one which many would happily eschew. It is a thankless and tedious operation that requires close, uninterrupted attention. Indeed, if one loses count an error or a slight hesitation might occur that would force one to start from scratch, and often to repeat the operation afresh, so as to guarantee the accuracy of the result. To this it should be added that it is generally necessary to perform the count on several different leaves in order to identify possible fluctuations, which can often be observed.

Such an irksome chore would certainly be carried out with more enthusiasm if the final product of the procedure were more immediately obvious, and if one could persuade oneself that the counting of lines may potentially yield useful information. However, bringing to mind Lao Tzu's subtle observation that 'a pot's usefulness lies in its emptiness', lines often seem to become more significant when they are missing rather than when they are in plain view. To attempt to estimate the number of lines borne by a specimen was a procedure that philologists experimented with very early on, in an effort to account for lacunae or other accidents of textual transmission. It should be pointed out that this approach more than once led to the said philologists rather recklessly exceeding the restricted limits that the state of codicology at the time would have permitted them to reach. Here, as a reminder to the reader, I shall cite only the speculations of Albert Curtis Clark in relation to the exemplar of Cicero's philosophical treatises, ${ }^{1}$ and those of Léon Herrmann on the tradition of Tibullus and other Latin poets. ${ }^{2}$ Finally, mention should also be made of a theory put forward by the same author, which was developed in $1932 .^{3}$

Translated from the French into English by Mark Livesey. Original published as Muzerelle, Denis (2009), 'Les propriétés arithmétiques de la linéation dans les manuscrits humanistiques', in Gazette du livre médiéval, 55: 20-31.

1 Clark 1918 (repr. 1969, 2004), chap. X, 182-363.

2 See in particular Herrmann 1967, 1015-1020.

3 Herrmann 1978.

Ә Open Access. (C) 2022 Denis Muzerelle, published by De Gruyter. (C) BY-NC-ND This work is licensed under the Creative Commons Attribution-NonCommercial-NoDerivatives 4.0 International License. https://doi.org/10.1515/9783110743838-009 
Other than the aforementioned cases, interest in the number of lines in manuscript volumes appears to be rather limited. To be sure, it is an element that combines with numerous others to define the material form of a book, and hence deserves to be included as part of any meticulous description. However, the immediate utility of such an observation is far from obvious. It can certainly be used to make an instant estimate of the text density on a page, but a similar result could be obtained by examining the relationship between the length of the text and the number of leaves it occupies. It can also be combined with the height of the justification so as to deduce the ruling unit, and thus to estimate the writing module. However, this is merely an approach that enables one conveniently to obtain a result which could be reached in other ways.

Rather than descending into numerological delirium, it is not easy to admit that the number of lines is purely the product of chance and does not contain a shred of usable information. Anyone who has ventured to systematically survey codicological data furnished in catalogues has not failed to come across, at one point or another, series of numbers that appear to possess a certain coherence, which subsequently inspire them to construct an explanatory hypothesis-until a new and totally incompatible series of numbers eventually emerges which sadly demolishes their pet theory.

Nevertheless, one can still formulate the hypothesis that if the same process is employed to set down the lineation of a certain number of manuscripts-even in those which exhibit a variable number of lines-the resulting ruling types will have certain arithmetic properties in common, such as being composed of an odd or even number of lines, or multiples of this or that number of lines.

Some other arithmetic properties can also be considered. Accordingly, if one imagines a scribe who starts out by establishing the justification at the top and bottom of the page, and then subdivides the resulting space in two, and then in turn divides these two spaces in two, and so on until the desired density is attained, the number of lines will necessarily assume the form $2^{\mathrm{n}}+1$. Of course, this is an artificial example, because such a protocol would only allow one to generate a very limited range of values (17, 33 or 65 , with regard to the 'useful portion'). This example is cited merely to illustrate the range of hypotheses that can be envisaged. Although it is not possible to determine all such hypotheses a priori, divisibility by various numbers remains the least difficult property to grasp, and so it is the one that we shall confine ourselves to examining here.

In order to verify the proposed hypothesis, the most natural propensity would be to apply statistical analysis in its most classical form. But in the present context we come up against a major problem: in order to be applicable, statistical analysis requires that each item/subject should belong to a category of the group under 
examination, and to one only. If, for example, we were to set about amassing statistics based on people's occupations, the case of an individual who practices palaeography in the morning and acrobatics in the afternoon would clearly pose a problem. If it were a unique case, it could be resolved in a one-off way, and would then become submerged in the overall mass of observations; but if it were a more widespread phenomenon or became more complex, one would have to forego applying the criterion of a defined profession. Now, this is the kind of situation that we are faced with here, because if one is dealing with multiples of 12 , one is also dealing with multiples of 2, 3, 4 and 6. A statistical approach is therefore only feasible if one limits oneself to considering solely prime numbers $(2,3,5,7,11$, etc.), and a quick glance will suffice to show that this restriction would prevent us from reaching any useful conclusions.

One must therefore proceed in an alternative way and return to the previous stage of the statistical approach-a stage where one is not yet concerned with the global distribution of occurrences, but instead only with the individual probability of each occurrence. Switching from a synthetical approach to an analytical one clearly will not simplify the task; in fact, it will considerably increase the number of calculations that would have to be performed. But that would be the only way to get around the problem, since by proceeding on a case-by-case basis it becomes irrelevant if a case happens to be a combination of two basic ones.

The underlying principle for this is as follows: by knowing the theoretical frequency $\boldsymbol{f}$ of an occurrence (or the frequency which one can consider normal), it is possible to calculate the probability of seeing it occur $\boldsymbol{x}$ times in a series of $\boldsymbol{n}$ random experiments. This value is directly provided by binomial law, which constitutes one of the fundamental basis the calculation of probabilities. By applying this model, one can compare each batch of $\boldsymbol{n}$ manuscripts exhibiting a total of $\boldsymbol{x}$ times the characteristic in question to a series of $\boldsymbol{n}$ random drawings from an urn in which the proportion of winning balls equals $\boldsymbol{f}$. One can then ask what the probability would be of observing such a result purely by chance, and draw conclusions based on the particular context concerned.

To interpret that probability, it must first and foremost be compared to a fixed reference point. Now, it has been demonstrated that in an experiment of this kind, the most likely result tallies with a mathematical expectation, namely with the product of $\boldsymbol{n}$ multiplied by $\boldsymbol{f}$. Even so, the probability of this result is not constant: it depends on $\boldsymbol{n}$ and on $\boldsymbol{f}$ by virtue of binomial law. One can therefore assess the more or less exceptional character of a given situation by comparing its probability to that of the mathematical expectation. Accordingly, a ratio of 0.5 indicates that one is in the presence of a situation more or less two times less probable than the 'normal' one; a ratio of 0.1 , ten times less probable, and so on. 
Here, a concrete example will help us to better understand the logic governing this procedure. The natural frequency of multiples of 5 is one in five, or 0.2. Therefore, in 20 manuscripts, the most likely situation that one will encounter is $20 \times 0.2=4$ where the lineation is a multiple of 5 , and the probability of obtaining this result is 0.218 . If the count is 6 , the probability of this different situation occurring is 0.109 , which is to say in a 0.5 ratio with the previous one. Such a scenario is therefore only two times less likely to occur, and so it would be prudent to consider that this may be the product of pure chance. However, if we are dealing with a count of 10 , the probability of obtaining such a result falls to 0.002 , and the ratio to 0.009 , indicating a probability of 107 times below the average. Thus, there is a less than one in a hundred chance that the phenomenon can be ascribed to pure chance, and one can legitimately enquire what this outcome means. To render the results easier to understand for readers unfamiliar with probabilities, it is the last value (in the example given above, 107) that will be used below to record the observed values, which will be arbitrarily given a plus (+) sign if higher than the mathematical expectation, and a minus (-) sign in the opposite case.

The question of what one must consider the 'normal' frequency still has to be cleared up. One's first inclination would be to compare it to the natural frequency, which has the advantage of being relatively easy to calculate: one number in two is a multiple of 2, one in three of 3, and so on. However, a quick analysis of the data immediately makes it clear that the frequencies observed across the entire corpus under investigation stray markedly from the 'natural' frequencies. They consist as follows (in percentages) (Tab. 1):

Divisibility frequencies

(tab. 1)

\begin{tabular}{|c|c|c|c|c|c|c|c|c|c|c|c|}
\hline Pr. & $2 n$ & $3 n$ & $4 n$ & $5 n$ & $6 n$ & $7 n$ & $8 n$ & $9 n$ & $10 n$ & $11 n$ & $12 n$ \\
\hline ca 25 & 50.0 & |33.3 & |25.0 & 20.0 & | 16.7 & |14.3 & 12.5 & |11.1 & | 10.0 & 9.1 & 8.3 \\
\hline 21.2 & 54.9 & 33.6 & 28.1 & 22.0 & 19.9 & 15.7 & |13.8 & 10.4 & 11.4 & 9.3 & 10.0 \\
\hline
\end{tabular}

$\mathrm{Nf}=$ natural frequency; $\mathrm{Of}=$ observed frequencies $-2 \mathrm{n}, 3 \mathrm{n}, \ldots=$ multiples of 2, of 3 , and so on

Tab. 1: Divisibility frequencies

Despite being low in terms of absolute values, these intervals exhibit some massive disparities in comparison to the probabilities generated by binomial law. On 
the other hand, given that we know that the manuscripts were made using several different techniques, it is of little help to learn that all these procedures taken as a whole lead to a bias in either this or that direction, without being able to know how to explain which of them is responsible for what. Conversely, the opposite approach, which aims to identify the consequences stemming from the use of different procedures, can make it possible to apprehend the overall situation. As a result, the frequencies to which it is most appropriate to refer are those that one observes in the corpus as a whole, which we can call the 'local norm'.

Prime numbers present a particular problem. As is well known, their distribution among all numbers is not consistent, since they gradually become scarcer as one ascends towards larger numbers. This irregularity is already quite noticeable within the small interval that we are dealing with here: 6 prime numbers can be counted between 6 and 29(30\%), 5 between 30 and 49(25\%), and 4 between 50 and $69(20 \%)$. The density of the distribution of prime numbers remains a mathematical conundrum which lacks a solution, so one can do no better than to adopt an average value and to factor in the phenomenon when interpreting results.

We have now reached a suitable point where questions can be posed about the codicological characteristics that are liable to have a direct impact on the practice of lineation, or which indirectly result in significant differences to it.

Needless to say, the particular ruling technique employed occupies the first rank. Even if the use of this expression-ruling technique-seems to have imposed itself among codicologists, it is not in fact very satisfactory, since it simultaneously refers to the tool employed to draw the ruling, the way in which the tool was handled, and the trace it left on the writing support. In actual fact, only the last aspect can be observed, since the other two are open to subjective interpretation, and are far from being unambiguous. The most important area of ambiguity concerns the techniques that leave an embossed impression (that is a sequence of ridges and furrows) on the support, which one can ascribe (with respect to later periods) either to the use of drypoint or of a ruling board. Often, only a close examination will enable one to distinguish between the two. Nevertheless, where embossed lineation is accompanied by a complete sequence of marginal pricking, one can be sure that it was drawn line-by-line, and therefore by drypoint. In the paragraphs that follow, these clear-cut cases are referred to using the term 'drypoint', whilst the others are referred to using the term 'blind (ruling)'.

The ruling type can also be suspected as having a direct influence on the number of lines. The distribution of vertical lines certainly exerts no influence, but the presence of overrun lines, either alone or in pairs, at the top or bottom of the lineation can exert a direct influence on the number of lines they frame. Here, layouts will be reported in accordance with the conventions set out by the 'universal 
formula': ${ }^{4}[0-0]$ for a total absence of overrun lines; [1-0] for the presence of a single overrun line at the top of the page but absent at the bottom, and so on.

By the same token, the use made of the ruling type-above or below top line [ATL, BTL], in accordance with the established nomenclature-should certainly be taken into account.

Finally, codex size is probably not without some significance-not because larger manuscripts make it possible to align a higher number of writing lines (the distribution of divisibility is not perceptible), but because a greater page height affords the artisan more flexibility to work. Indeed, one can imagine that the scribe would have chosen the number of lines (if it were not stipulated beforehand) in the following way: first he set the order of magnitude (in relation to his script and the size of the manuscript), then he established the precise number of lines so as ensure compatibility with the line-tracing method. In such circumstances, large manuscripts offer greater flexibility than is the case with smaller ones. The fact that the extent of this flexibility is most likely not in harmony with the optimal value-given that it is easier to broaden the script and increase the distance between lines than to do the opposite-is irrelevant in this context.

To put this hypothesis to the test, one must have at one's disposal a collection of data that censuses a large number of manuscripts by including all the aforementioned parameters, and above all one which furnishes a 'standardised' number of lines, rather than minimum-maximum values. Albert Derolez's work on the codicology of Humanistic manuscripts ${ }^{5}$-a resource which remains largely underexploited-is beyond doubt the only source that draws together the requisite conditions.

The application of the foregoing defined protocol to our corpus yielded the results that are presented in Tab. 2.

4 Muzerelle 1999.

5 Derolez 1984. 


\section{$\underline{N b} \underline{P r} . \underline{2 n} \quad 3 n \quad 4 n \quad 5 n \quad 6 n \quad 7 n \quad 8 n \quad 9 n \quad 10 n \quad 11 n \quad 12 n \quad 13 n \quad 5 / x /$}

\begin{tabular}{|c|c|}
\hline \multicolumn{2}{|l|}{ Size } \\
\hline |Large & |388, 38 -1.9, $1.3,1.1,1.0,1.2,1.0,1.9,1.0,2.3,1.0,1.0,1.0,-13.3,29.0$ \\
\hline |Medium & $\left.\left.\left.\left.\left.\left.\left.\left.\left.\left.\left.\left.\left.\left.\left.\right|^{384}\right|^{2.1}\right|^{-1.0}\right|^{-1.2}\right|^{1.0}\right|^{-1.2}\right|^{-1.5}\right|^{-1.1}\right|^{-1.2}\right|^{2.3}\right|^{-1.6}\right|^{1.0}\right|^{-6.9}\right|^{1.5}\right|^{22.6}$ \\
\hline |Small & 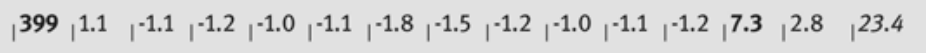 \\
\hline \multicolumn{2}{|c|}{ Overrun lines } \\
\hline । $0-0$ & 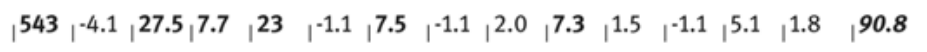 \\
\hline |1-0 & $\left.\left.\left.\left.\left.\left.\left.\left.\left.\left.\left.\left.\left.\left.\left.\right|^{105}\right|^{276}\right|^{-60}\right|^{1.0}\right|^{-3.7}\right|^{-1.1}\right|^{-1.1}\right|^{-1.0}\right|^{-3.2}\right|^{1.3}\right|^{1.0}\right|^{-1.1}\right|^{-1.0}\right|^{-1.3}\right|^{353.6}$ \\
\hline$\left.\right|^{1-1}$ & $\left.\left.\left.\left.\left.\left.\left.\left.\left.\left.\left.\left.\left.\left.\left.\right|^{165}\right|^{1.6}\right|^{-1.7}\right|^{-1.0}\right|^{-1.1}\right|^{-1.5}\right|^{-1.0}\right|^{-1.4}\right|^{2.8}\right|^{-1.3}\right|^{1.4}\right|^{-1.8}\right|^{-1.1}\right|^{-1.0}\right|^{18.9}$ \\
\hline$\left.\right|^{2-0}$ & $\left.\left.\left.\left.\left.\left.\left.\left.\left.\left.\left.\left.\left.\left.\left.\right|^{12}\right|^{-1.1}\right|^{2.4}\right|^{-1.1}\right|^{1.1}\right|^{3.5}\right|^{1.2}\right|^{-1.0}\right|^{1.9}\right|^{-1.4}\right|^{11.5}\right|^{1.0}\right|^{-1.3}\right|^{-1.1}\right|^{29.8}$ \\
\hline$\left.\right|^{2-1}$ & | $8.0,1.0,1.0,-\left.\left.\left.\left.\left.\left.\left.\left.\left.\left.\left.7.3\right|^{1.3}\right|^{-1.0}\right|^{-1.7}\right|^{1.5}\right|^{1.0}\right|^{-1.1}\right|^{-1.0}\right|^{1.0}\right|^{-1.1}\right|^{-3.0}\right|^{23.1}$ \\
\hline$\left.\right|^{2-2}$ & $\left.\left.\left.\left.\left.\left.\left.\left.\left.\left.\left.\left.\left.\left.\left.\right|^{338}\right|^{1.0}\right|^{-2.0}\right|^{-4.2}\right|^{-14.5}\right|^{1.2}\right|^{-3.3}\right|^{2.0}\right|^{-3.1}\right|^{-5.4}\right|^{-3.6}\right|^{2.3}\right|^{-2.0}\right|^{-1.0}\right|^{45.5}$ \\
\hline
\end{tabular}

Utilisation (above/below top line)

,ATL

| $\left.\left.\left.\left.\left.\left.\left.\left.\left.\left.\left.\left.\left.\left.452\right|^{-1.5}\right|^{6.9}\right|^{3.0}\right|^{28.7}\right|^{1.0}\right|^{14.6}\right|^{-2.3}\right|^{7.2}\right|^{17.3}\right|^{2.0}\right|^{-1.2}\right|^{85}\right|^{-2.5}\right|^{173.1}$

,BTL

| $\left.\left.\left.\left.\left.719\right|^{1.8}\right|^{-3.0}\right|^{-1.5}\right|^{-10.4}\right|^{1.0},-3.7,1.4,-\left.\left.\left.\left.\left.\left.2.7\right|^{-5.4}\right|^{-1.2}\right|^{1.0}\right|^{-18}\right|^{2.0}\right|^{53.1}$

Technique

I Ink $\left.\left.\left.\left.\left.\left.\left.\left.\left.\left.\left.\left.\left.\left.\left.\quad\right|^{492}\right|^{-2.1}\right|^{12.5}\right|^{12}\right|^{10.6}\right|^{1.0}\right|^{19.7}\right|^{-1.0}\right|^{-1.0}\right|^{7.4}\right|^{3.5}\right|^{1.1}\right|^{4.7}\right|^{-1.1}\right|^{77.8}$

|Leadpoint $\quad \quad$ 75 $\quad$ | $\left.\left.\left.\left.\left.\left.\left.\left.\left.\left.\left.\left.\left.4.4\right|^{-104}\right|^{1.3}\right|^{-9.0}\right|^{1.0}\right|^{-2.6}\right|^{-3.0}\right|^{1.0}\right|^{1.0}\right|^{-1.1}\right|^{-1.2}\right|^{-1.3}\right|^{1.0}\right|^{131.8}$

|Drypoint $\quad$ 252 $\left.\left.\left.\left.\left.\left.\left.\left.\left.\left.\left.\left.\left.3.8\right|^{-2.0}\right|^{-3.4}\right|^{-1.1}\right|^{1.0}\right|^{-1.9}\right|^{-1.1}\right|^{1.6}\right|^{-3.0}\right|^{-1.0}\right|^{1.0}\right|^{1.0}\right|^{-1.0}\right|^{23.0}$

|Blind $\left.\left.\left.\left.\left.\left.\left.\left.\left.\left.\left.\left.\left.\left.\quad 352\right|^{-1.0}\right|^{-1.0}\right|^{-2.3}\right|^{-2.3}\right|^{1.0}\right|^{-1.5}\right|^{1.7}\right|^{-1.1}\right|^{-1.6}\right|^{-1.9}\right|^{-1.9}\right|^{-2.8}\right|^{1.6}\right|^{21.6}$

Pricking system

|None $\quad$ 676,1.0,1.1 $\left.\left.\left.\left.\left.\left.\left.\left.\left.\left.\left.\left.\right|^{1.3}\right|^{-1.3}\right|^{1.0}\right|^{1.2}\right|^{-1.1}\right|^{-1.0}\right|^{1.0}\right|^{1.4}\right|^{-1.3}\right|^{-1.1}\right|^{1.4}\right|^{15.2}$

|Complete system | $\left.\left.\left.\left.\left.\left.\left.\left.\left.\left.\left.\left.\left.\left.278\right|^{2.5}\right|^{-5.9}\right|^{-2.4}\right|^{-1.8}\right|^{1.1}\right|^{-3.3}\right|^{-1.0}\right|^{1.3}\right|^{-4.7}\right|^{-1.1}\right|^{1.3}\right|^{-1.1}\right|^{-1.0}\right|^{28.6}$

|Vertical $\left.\left.\left.\left.\left.\left.\left.\left.\left.\left.\left.\left.\left.\left.\quad \mathbf{1}^{185}\right|^{-2.2}\right|^{1.4}\right|^{2.1}\right|^{3.8}\right|^{1.2}\right|^{1.9}\right|^{1.0}\right|^{-1.0}\right|^{5.5}\right|^{1.0}\right|^{1.0}\right|^{2.6}\right|^{-1.3}\right|^{26.1}$

| Single $\left.\left.\left.\left.\left.\left.\left.\left.\left.\quad\right|^{35}\right|^{-1.1}\right|^{3.6}\right|^{-1.5}\right|^{12.5}\right|^{-2.9}\right|^{1.0}\right|^{7.8}\right|^{1.0}, 1.1,\left.\left.\left.\left.\left.\right|^{-3.1}\right|^{1.2}\right|^{1.6}\right|^{1.0}\right|^{39.4}$

Tab. 2: Divisibility of lineation: compared to the probable norm (inverse)

An initial observation can immediately be made by looking at the raw data: the situation is far from being neutral or 'flat'. Even if many figures lie within bounds that can be ascribed to pure chance, many others denote phenomena that are clearly significant. At the same time, one can see that different lines present similar 'profiles', 
which is to say that in the same columns a few lines distant one observes minimums and maximums that are comparable. This is a direct reflection of the lack of independence between techniques belonging to distinct categories-for example, ink lineation enjoys an overwhelming majority in the layout type [0-0]. Tab. 3 presents the co-occurrences observed among all the modalities.

Co-occurrence of modalities in B(ig), M(edium) and $\mathrm{S}$ (mall) manuscripts

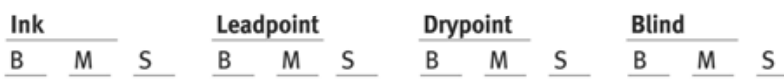

\begin{tabular}{|c|c|c|c|c|c|c|c|c|c|c|c|c|}
\hline ATL & |45 & $\left.\right|^{57}$ & $\left.\right|^{56}$ & $1^{7}$ & 9 & $1^{5}$ & $\left.\right|^{1}$ & $1^{4}$ & $1^{3}$ & $1^{5}$ & $1^{7}$ & $1^{5}$ \\
\hline BTL & 57 & 81 & 116 & $1^{2}$ & $1^{4}$ & 10 & $1^{2}$ & - & $\left.\right|^{1}$ & |24 & 18 & 24 \\
\hline
\end{tabular}

1-0

IATL $\left.\left.\left.\quad\right|^{3} \quad\right|^{9} \quad\right|^{3} \quad-\left.\left.\left.\left.\left.\left.\left.l^{2} \quad\right|^{3} \quad\right|^{8} \quad\right|^{3} \quad\right|^{1} \quad\right|^{1} \quad\right|^{2} \quad\right|^{3}$

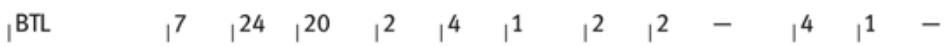

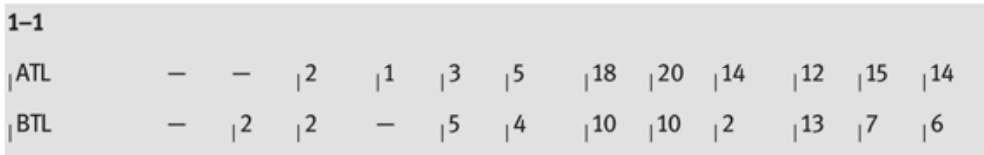

2-0

|ATL

|BTL

$$
-\left.\left.\left.\right|^{1}\right|^{1}\right|^{2}-\left.\right|^{2}-\left.\right|^{2}-\left.\left.\right|^{2}\right|^{1}-
$$

2-1

ATL

BTL

$$
\begin{array}{ccccccccccccc}
- & - & - & - & - & - & \left.\right|^{2} & \left.\right|^{2} & - & - & - & - \\
- & - & - & - & - & - & - & \left.\right|^{1} & \left.\right|^{1} & \left.\right|^{1} & - & \left.\right|^{1}
\end{array}
$$

2-2

।ATL

|BTL

$$
\begin{array}{llllllllllll}
।^{1} & \left.\right|^{1} & - & - & - & \left.\right|^{1} & \left.\right|^{23} & \left.\right|^{20} & \left.\right|^{13} & \left.\right|^{23} & \left.\right|^{9} & \left.\right|^{10} \\
।^{1} & \left.\right|^{2} & \left.\right|^{1} & ।^{2} & - & \left.\right|^{1} & ।^{42} & \left.\right|^{21} & \left.\right|^{23} & \left.\right|^{64} & \left.\right|^{35} & \left.\right|^{45}
\end{array}
$$

Tab. 3: Co-occurrence of modalities in B(ig), M(edium), S(mall) manuscripts 
This situation is not easy to analyse, since each phenomenon observed simultaneously in two correlated lines makes it necessary to carry out further tests in order to determine which of the two techniques exerts an effect on the other.

This question immediately arises with respect to the two lines where we can see that the strongest figures gather in the same columns: the first relates to ink lineation; the second to ruling types without overrun lines ([type 0-0]). By examining, respectively, what happens in all the ink rulings and in the type [0-0] ruling types drawn by other means (it would take too long to present the relevant figures here), one can establish that it is the use of ink which is largely responsible for these gaps in the expected norm, and not vice versa.

Now, the cardinal characteristic that distinguishes ink lineation from other techniques is the fact that it is likely to have been executed using a so-called 'rake', as a certain number of specific observations show. ${ }^{6}$ This helps us to recognise in this technique the reasons for the deviations from the expected norm.

In order to verify this assertion, it is necessary to examine the arithmetic properties which are allied to the use of a rake. These are easy to demonstrate. If the rake has an even number of tines, it will produce, in one fell stroke, an even number of lines. If its tines are of an uneven number and the lines drawn any number of times on the page, one time out of two it will produce an even number of lines. All in all, the even numbered lineations will always be in the majority, and that is precisely what we observe here.

But we can stretch our reasoning even further: if the choice between an even numbered rake and an odd numbered one is governed entirely by chance, the average share for each of the two types will be 50\%, resulting in a final tally of $75 \%$ even-numbered lineations and 25\% uneven. What, then, do we find in our corpus? The manuscripts ruled in ink total 492, from which we must exclude the 93 cases that correspond to a prime number, and which therefore cannot be included within the envisaged framework (we shall meet with them again in due course). Of the 399 that remain, 295 present an even-numbered lineations, and 104 an uneven one, being $73.9 \%$ and $26.1 \%$ respectively. It is hard to imagine a better match of the facts with the theory. With respect to ink rulings, one can only deduce that there was widespread and massive use of rakes in which the number of tines was not standardised.

This conclusion is not entirely unexpected: as we have already said, the use of rakes has long been demonstrated through the archaeological approach. But what one is often forced to merely suspect (in the absence of hard evidence) is now confirmed in a dramatic way. The main evidence is provided by the pricking

6 See, in particular, Gumbert 1986. 
system, a single lateral pricking (System 4, according to Derolez's classification) can be considered indicative of the use of a rake. But alas, this is a rather vulnerable indicator, since it can be eliminated through trimming or is impossible for the observer to discern. Only $7.1 \%$ of manuscripts ruled in ink exhibit such pricking, but $91.7 \%$ of these lack a complete system of lateral pricking, which indisputably suggests the drawing of lines one-by-one. Analysis of the lineation reveals that the vast majority of these were ruled using a rake.

Another issue that draws our attention concerns the [1-0] ruling types. This type is characterised by a significant sparsity of even numbers. The relationship inclines necessarily in this direction, because no algorithm exists that enables us to consistently generate prime numbers, or to generate a predominance of them. Evidence for this can be seen in the huge effort mathematicians have to make to discover, by trial and error, new prime numbers for data encryption purposes. And what is true of large numbers is also true of small ones, apart from the fact that a change in scale reduces the amount of experimentation required.

The foregoing observation leads one to think that the sparsity of even numbers is, so to speak, 'accidental', and not a direct result of the multiple ruling method. All the evidence suggests that it is caused by the addition of a supplementary line-the initial overrun line-to a lineation generated through a procedure which largely produces lines in even numbers.

The use of a rake provides the most satisfactory explanation because, on the one hand, it predominantly generates even-numbered lineations (as shown), whilst on the other it can only draw lines of a consistent length, so that the overrun line must necessarily pre-exist and be added to the tally. Strictly speaking, this interpretation can only partially explain the phenomenon, since manuscripts ruled in ink only in fact represent two cases in three (39 out of 62, or 63\%). However, such a proportion is perfectly adequate to ascertain the overall situation.

We can now return to the case of the manuscripts with a prime number of lines and without overrun lines, which we had previously left aside. What we have just established allows us to suppose that in actual fact these manuscripts do have an overrun line, which can we term 'an overrun line of reduced length' or a 'crypto-overrun line'-in other words, a writing line which is the same length as the others but was traced prior to the execution of the actual ruling drawn by means of a rake.

Naturally, one would be pleased to be able to furnish solid proof for these hypotheses by analysing the case of ruling types exhibiting three overrun lines-because when they are of an even number (two or four, configured as [1-1], [2-0], or [2-2]) the phenomenon does not occur. Unfortunately, the [2-1] types are 
too rare (only 11 examples) to have any statistical value; furthermore, some of them are not ruled in ink.

The second major phenomenon which can be gleaned from Tab. 2 concerns the use of 'above top line' (ATL) or 'below top line' (BTL) ruling, where the distribution departs significantly from that which pure chance should allow.

Interpretation of this phenomenon is far less immediate and requires that one first establishes statistics for the correlation between the parity in lineation and its use across the overall corpus. In order to avoid needlessly complicating matters, the following table only presents figures in relation to BTL. Regarding the opposite use (i.e. ATL), it is simply the mirror image of that presented in the table.

Overall, the use of BTL predominates in the corpus viewed as a whole, and this preference is appreciably stronger in the uneven-numbered lineations compared to the even-numbered ones. However, this situation changes if one considers one-byone the subsets corresponding to the various techniques taken into account up till now (Tab. 4).

\begin{tabular}{|c|c|c|c|c|c|}
\hline \multicolumn{5}{|c|}{ Correlation between odd/even numbers and utilisation BTL } & \multirow{2}{*}{$\begin{array}{r}\text { (tab. 4) } \\
\text { Odd/Even } \\
\text { difference }\end{array}$} \\
\hline & All & $\begin{array}{l}\text { Difference in } \\
\text { comparison to } \\
\text { the entire group }\end{array}$ & $\begin{array}{l}\text { Odd } \\
\text { lineation }\end{array}$ & $\begin{array}{l}\text { Even } \\
\text { lineation }\end{array}$ & \\
\hline Entire group & |61.4\% & - & $65.4 \%$ & ¡58.1\% & $1+7.3 \%$ \\
\hline Ink & |64.0\% & $+2.6 \%$ & , $67.5 \%$ & |61.7\% & $1+5.8 \%$ \\
\hline | Leadpoint and drypoint & , $48.6 \%$ & $-12.8 \%$ & |54.1\% & 142.7\% & $+11.4 \%$ \\
\hline |Leadpoint & ן $52.0 \%$ & $1-9,4 \%$ & $48.9 \%$ & ,57.1\% & $\left.\right|^{-8.2 \%}$ \\
\hline |Drypoint & $1^{47.6 \%}$ & $-13.8 \%$ & |56.0\% & $39.5 \%$ & । $+16.5 \%$ \\
\hline |Blind & |69.7\% & । $+8.3 \%$ & 175.0\% & |65.3\% & $1+9.7 \%$ \\
\hline | Type 0-0 & |62.4\% & । $+1.0 \%$ & $66.0 \%$ & $60.1 \%$ & $1+6.0 \%$ \\
\hline | Type 1-0 & |63.8\% & । $+2.4 \%$ & $62.9 \%$ & | $65.1 \%$ & $1^{-2.2 \%}$ \\
\hline | Type 1-1 & $37.0 \%$ & $\mid-24.4 \%$ & $46.9 \%$ & $27.4 \%$ & । $+19.5 \%$ \\
\hline | Type 2-2 & | $70.1 \%$ & $1+8.7 \%$ & ,73.6\% & | $66.9 \%$ & $1+6.8 \%$ \\
\hline
\end{tabular}

Tab. 4: Correlation between odd/even numbers and utilisation BTL ('below top line') 
If one does not dwell on minor variations, one will more than anything notice the following phenomena become clear: the predominance of the use of BTL diminishes considerably in parallel with the two techniques that involve the ruling being drawn line-by-line, regardless of whether this is done using drypoint or leadpoint, and the tendency is even reversed, albeit very slightly, when the latter technique is employed. But the effect on the preference between uneven- and even-numbered lineation is radically different in the two cases: uneven-numbered lineation is preferred when ruling is drawn in drypoint, whereas the advantage shifts to even-numbered lineation wherever leadpoint is used.

One observes the 'echo' of this phenomenon, considerably amplified, in the [1-1] types. It would be tempting to conclude that this is due to the effect of the line-by-line techniques, which are largely predominant in this group (54\%), and all the more so given that almost all of the balance (43\%) is composed of blind ruling, in which a not negligible proportion (albeit indefinable) is attributable to drypoint. Nevertheless, this interpretation is contradicted by the case of the [2-2] types, where drypoint and blind ruling are also over-dominant-but in opposite proportions: $43 \%$ and 55\% respectively-without producing the same effects. Therefore, it is the presence of a single overrun line at the top and the bottom of the justification which is responsible for producing this situation.

To summarise: the [1-1] type brings about an increasing scarcity of the use of BTL and its focus on uneven-numbered lineation. Thus, there exists a natural link between the two characteristics, whose mechanism, for now, remains obscure.

The preceding material, which we have intentionally confined to addressing only the most immediate aspects of the problem, obviously does not purport to have fully exhausted the subject matter. There is undoubtedly more to be learned through the arithmetic analysis of the number of lines in manuscript ruling, but any endeavour to do so will certainly prove to be a considerably more arduous task. All the same, this preliminary exploration should have succeeded in highlighting the utility of carrying out accurate censuses of the number of lines in manuscripts, and above all of recording the resultant observations in a useful form.

One must commend the zeal with which Jean Mallet and André Thibaut, in their Manuscrits en écriture bénéventaine de la Bibliothèque capitulaire de Bénévent, ${ }^{7}$ provide meticulous details of lineation, recording variations observed on a leaf-by-leaf basis. However, one obviously should not impose a similarly

7 Mallet / Thibaut $1984\left(1997^{2}\right)$. 
stringent model on all cataloguers. At most, one should encourage them to report their observations in a readily interpretable way, above all by abandoning the erroneous notion that a piece of information is more pertinent when it is presented in a strict numerical formula than when it is written in a discursive manner. An indication such as '48 lines, barring exceptions' is a thousand times preferable to '48-50 lines'-or even '48-72 lines', if the exception constitutes an anomaly: only collectors of oddities will feel aggrieved. In fact, if the cataloguer can take responsibility for ascertaining the figure which he deems to best coincide with reality, the statistician remains powerless when faced with a value expressed in the form of a range, whose precise extent is impossible for him to surmise. To be sure, in this regard we will have to wait for cataloguing practice to undergo a certain amount of reform - which undoubtely will take a long time at the end-before the research embarked upon here can be extended to other sectors of the codicological domain.

\section{References}

Clark, Albert Curtis (1918), The Descent of Manuscripts, Oxford: at the Clarendon Press [repr. in 1969, 2004].

Derolez, Albert (1984), Codicologie des manuscrits en écriture humanistique sur parchemin, I. Texte, II. Catalogue, Turnhout: Brepols (Bibliologia, 5-6).

Gumbert, Johan Peter (1986), 'Ruling by Rake and Board: Notes on some Late Medieval Ruling Techniques', in Ganz, Peter (ed.), The Role of the Book in the Medieval Culture, Turnhout: Brepols (Bibliologia, 3), 41-54.

Herrmann, Léon (1967), 'La règle des dix-huit vers et les livres XIII et XIV de Martial', in Latomus, 26: 1015-1020.

Mallet, Jean / Thibaut, André (1984 [1997²]), Manuscrits en écriture bénéventaine de la Bibliothèque capitulaire de Bénévent, Paris: CNRS [Turnhout: Brepols 1997²].

Muzerelle, Denis (1999), 'Pour décrire les schémas de réglure: une méthode de notation symbolique applicable aux manuscrits latins (et autres)', in Quinio. International Journal on the History and Conservation of the Book, 1: 123-170. 
